\title{
The design and implementation of intelligent mobile message Alarming system
}

\author{
Faying $\mathrm{Li}^{1, \mathrm{a}}$, Shanquan $\mathrm{Hu}^{2, \mathrm{~b}}$ \\ ${ }^{1}$ Department of computer science \\ Xiang Nan University,Chen Zhou, China \\ ${ }^{2}$ Department of computer science \\ Xiang Nan University,Chen Zhou, China \\ alifaying888@163.com, bctzwhsq@163.com
}

Keywords: monolithic; Q2403A; short message; alarming system

\begin{abstract}
At present, housing security is essential to people's daily life, so the application of monitor system is becoming more and more common. According to the background, the schema of transmission of alarming information to mobile call is introduced .The alarming system is composed of monolithic module and mobile call module. After the alarming signals is sent to the monolithic, then the monolithic sent the signal to mobile call module Q2403A through AT instruction .Then the mobile call module sent message to designated call number. Security management office can deal with emergency situation through finding the useful information displayed on screen of the mobile call.
\end{abstract}

\section{Introduction}

The business does not need to create a specific link, only need send short news to a target cell phone. Owing to its simplicity, cheap expenses, the wide overlay scope, GSM short messages business is widely used.

The design and realization of GSM short message system is described in this paper, a concrete design schema including hardware connection and software design is given. Last, the system alarming function is realized. We realize the short message alarming system by using monolithic so that external circuit is decreased, the whole system performance can be increased including simple circuit, stable function. In the following, we introduce each part in detail.

\section{Architecture of the system}

The system's hardware is mainly composed of the STC11 L04 E monolithic, Q2403 A cell phone modular and the power converter .The architecture of system is shown in Fig.1.



Fig.1 Architecture of system

The electric voltage of cell phone modular is 3.3 volts, so when we choose monolithic we think the Q2403 is more useful because its work electric voltage is a $3.3 \mathrm{~V}$, so we choose the STC11L04E as our work monolithic, as a result, this can simplified the electric circuit, also make the electric voltage consistent at the time of their communication, it is not necessary to electric voltage conversion again. Secondly, There is $1 \mathrm{~K}$ Byte EEPROM in the STC11 L04 E monolithic, the big storage location can 
be used to save a customer phone number, so we don't need to connect an external EEPROM chip, this can simplify the whole electric circuit of system.

Introduction of Power.The design of the power is relation to the stability of the whole system. In order to increase the stability, we connect a 12-volt power through a 12-volt power adapter. The whole system only needs a 3.3-volt work voltage. Because the cell phone modular Q2403 has a request of stability to electric voltage, so we choose LM2596 chip that has steady voltage, we once try to use LM1085,it can not reach our requirements because if we choose LM1085,the cell phone modular has not signals. In order to get more effective stable voltage, in addition, we use VC1 and VC6 that have enough electric capacity. The principle diagram of the power is shown in Fig.2.

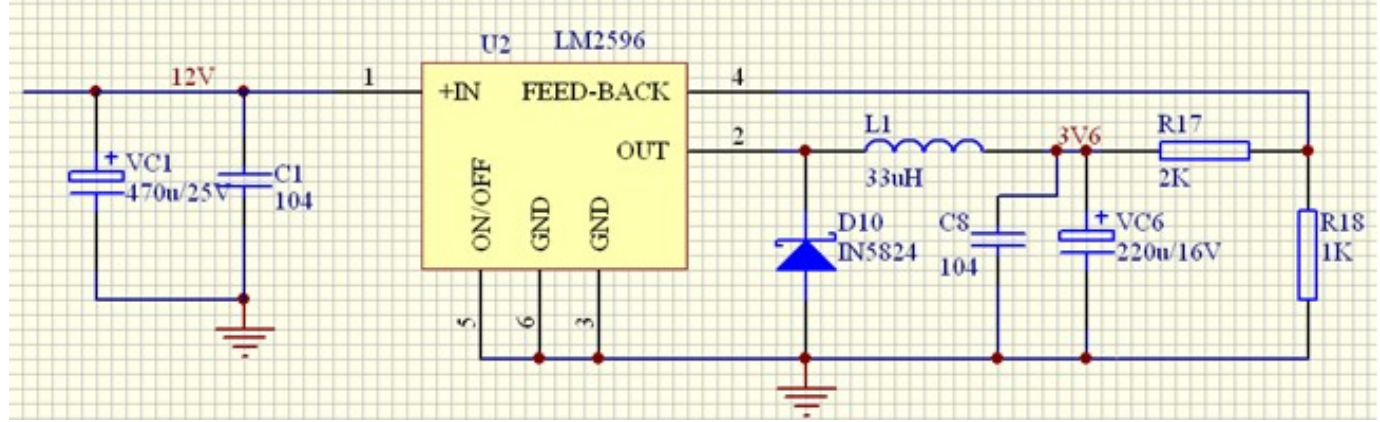

Fig.2 the principle of power electric modular

Introduction of monolithic. The monolithic part is the whole control core of system, we need at least a serial port, in addition, we need choose inner EEPROM, the flash that has 3k mainly used to storage code. Considering this, we choose STC11L04E, it is suitable.Secondly, and STC11L04E is convenient for burning to record program, so it is convenient for debugging the program in development process.

STC11 L04 E is the product of MCS-51 series monolithic, it functions as 8051 monolithic [1][2].The STC11 L04 E monolithic includes CPU, ROM, RAM, timer/counter, parallel interface, serial interface and interruption system...etc. At the same time, it has three buses such as data bus, address bus and control bus. we introduce the function of each part in detail:

1) CPU

The CPU(CPU) is the core component of the whole monolithic, it is a 8-bit processor that can handle 8-bit binary data or code, CPU is responsible for controlling, directing and adjusting the work of whole system, along with completing operation and control input and output operations.

CPU mainly consists of arithmetic/logic unit and control unit.

2) Full Duplex serial port

STC11 L04 E has 2 built-in full duplex serial ports, used for data transmission with other devices. We generally use one set to download program, and the other set is used for communicating with other external devices. These 2 sets full duplex serial port use different pin feet, there is no difference on the function.

There are two independent data line in STC11 L04 E that is TXD and RXD. It allows a data to deliver toward two contrary directions at the same time. Generally send data while corresponding through TXD port and receive data through RXD.

EEPROM.The EEPROM of STC11 L04 E has EEPROM inside the slice that can wipe out more than 100,000.Used for depositing parameter; these parameters can't throw to lose even if the power is turned off.

The structure of monolithic has two kinds. One is Harvard structure that is used to separate program from data, the other sis Princeton structure that is used for program storage and data storage and is used widely. MCS-51 of INTEL uses Harvard structure and the latest production of 16-bit MCS-96 serial uses Princeton structure.

The internal structure of MCS-51 serial is shown in Fig.3: 


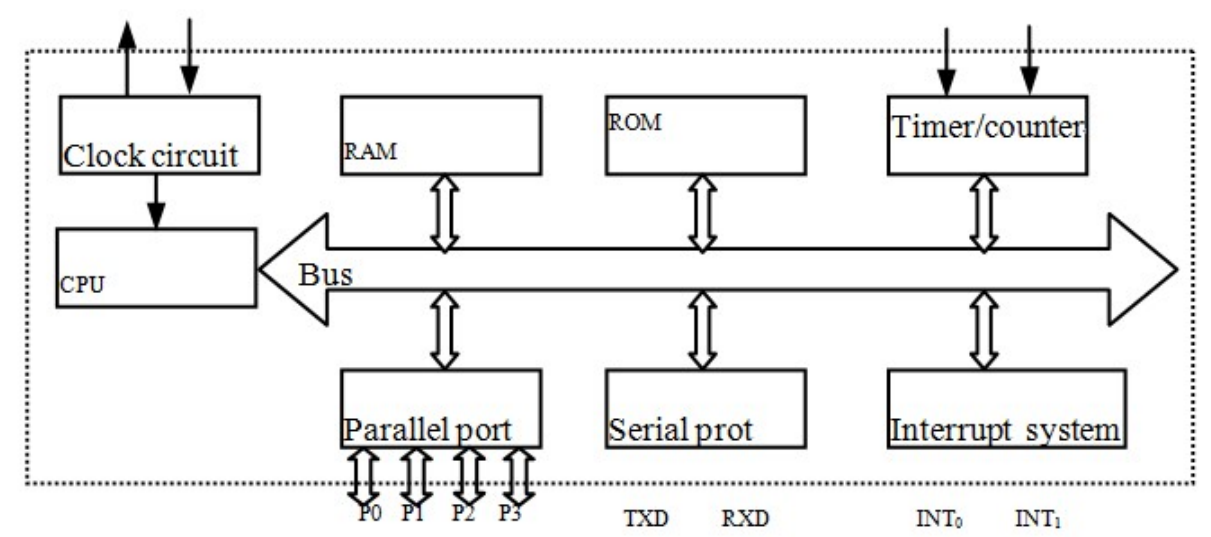

Fig.3 the internal structure of MCS-51

Monolithic receives voice signals from voice control sensor. When the power is turned on ,there is a high electrical level electricity add on P10,then when there is voice signal, voice control sensor send a low electrical level electricity, monolithic run short message sending program according to the receiving signal. Then monolithic communities with cell phone modular through TX2 and RX2.In there used to download program codes to monolithic.

Cell phone modular.Q2403 is a kind of cell phone modular with very strong functions, which has listening function, calling phone function receiving function and sending short message function in this paper, we only use its receiving function and sending message function. In compared to other kinds of products,Q2403 A cell phone modular has advantage of strong receiving function, and its signal is getting more stable so that can avoid causing message's sending out the circumstance of failure because of cellular phone network because of weak signal.

The GSM|GPRS modular of Q2403 support dual-band EGSM|GPRS 900 Mhzs|1800 MHz mode, support AT instruction, the short news function is complete.Q2403 A modular has general interface of 60 pin feet, integrate an application product very easily; Have SIM card confirmation, power supply, clock, reset and the SIM card interface, including SIMVCC, SIMRST, SIMDATA, SIMCLK and SIMPRES etc.. Moreover, Q2403 modular provides a 6-line serial correspondence interface based on V24 protocol including TX and RX, RTS and CTS, DTR and DSR; Provide DCD and RI signal to connect at the same time, last Power ON telecommunication| OFF and reset signal RST. the modular normal work requests to provide two road the powers, among them, VBATT provides to the RF part, the electric voltage scope is a $3.3 \mathrm{Vs} 4.5 \mathrm{Vs}$, the biggest power for the $2 \mathrm{Ws}$; VDD provide to take part for radical, the electric voltage scope is a $3.1 \mathrm{Vs} \sim 4.5 \mathrm{Vs}$, the biggest power is 0.5 Ws. We make through RX2 and TX2 with monolithic communication, monolithic send message contents and target cell phone number that need to be sent out to As to the Q2403 through an AT instruction, Q2403 feedback the result produced by short message modular to the monolithic. the principle of cell phone modular is shown in Fig.4

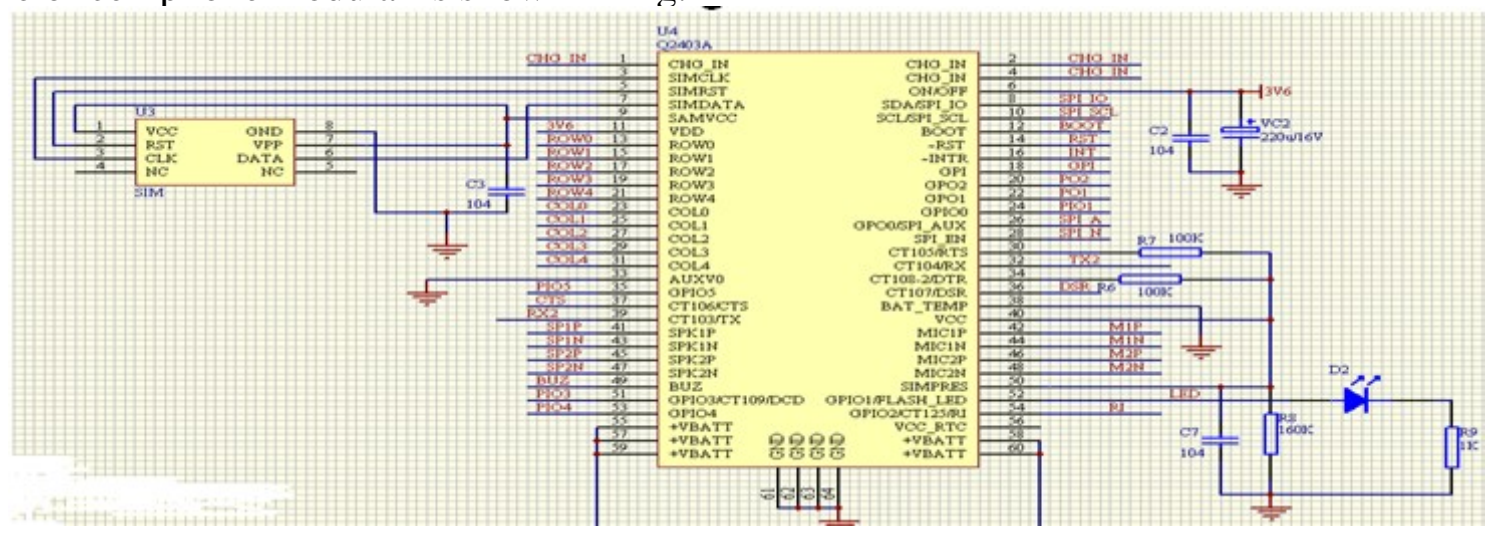

Fig.4 principle diagram of cell phone modular 
STC11 L04 E monolithic has 51 inside pit can use MCS-51 assembler language to program, it also supports C51 language, we use universal KEIL C to compile program[4][5]. Software structure this system is mainly divided into two big parts: main procedure and interruption sub-procedure. The main procedure does system beginning, including constitution of monolithic, interruption beginning, serial port constitution (baud rate, interruption allow etc.), constitution of default system data (such as positional notation clear zero, obtain a target cell phone number etc.) etc... Then wait the message of alarming arrival or the arrival of short message including parameter of modifying target cell phone number. Because when the message of useful modification target cell phone number arrives and need monolithic immediately respond to, just can receive a message correctly, so we use interruption method instead of serial receiving. The flow chart of main procedure is shown in Fig.5:

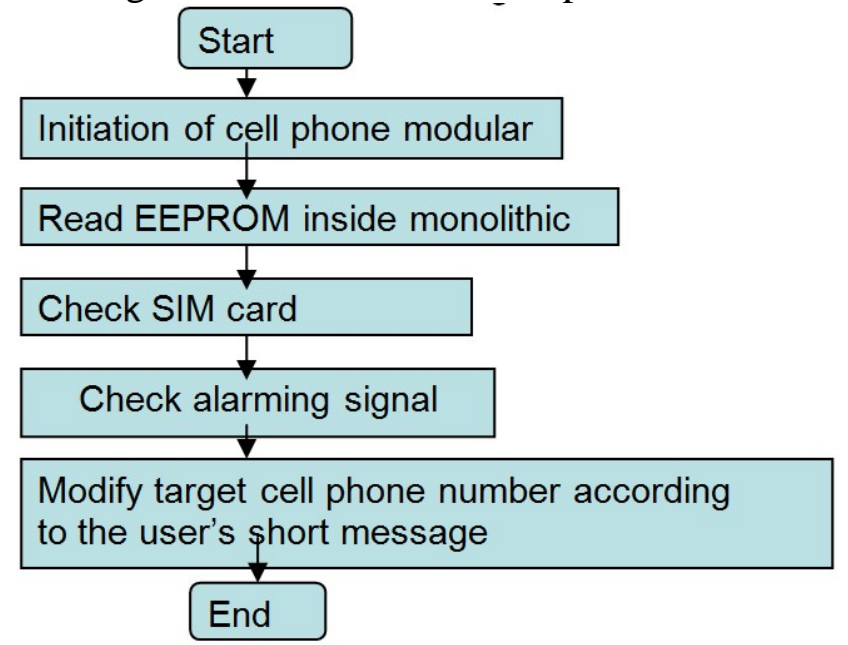

Lord procedure process is as follows:

When alarming signals are generated, short contents are sent to cell phone number that saved in the EEPROM of monolithic, so cell phone number is burned in EEPROM of monolithic when we record data and burn program, the processing is very convenient. When the program starts, the cell phone number saved in EEPROM is read, the readable function is as follows:

\|Read one byte, adjust to need to open IAP function before using, entrance: DPTR= byte address, return: $A=$ readable byte

INT8UByte_Read (INT16U add)

\{

IAP_DATA $=0 \times 00$;

IAP_CONTR = ENABLE_ISP; |Open IAP function, setting Flash operation waiting time

IAP_CMD $=0 x 01 ;||$ IAP $\mid$ ISP $\mid$ byte reading instruction of EEPROM

my_unTemp16.un_temp16 = add;

IAP_ADDRH = my_unTemp16.un_temp8 [0]; ||setting 8 high addresses of target unit address

IAP_ADDRL = my_unTemp16.un_temp8 [1]; ||setting 8 low addresses of target unit address

$\| \mathrm{EA}=0$

IAP_TRIG $=0 \times 5$ A; $\|$ Send 5 Ah first, then send the A5

IAP_TRIG = 0xA5; ||Finish sending A5 h, ISP|IAP is immediately triggered to start nod_();

$\| \mathrm{EA}=1$;

IAP_Disable (); losing IAP function and make CPU be safe status,

$\|$ A continuous IAP operation completion suggests to close IAP function after, don't need to be closed each time

Return (IAP_DATA);

\}

Using this function and then acquire the data in EEPROM, get the user's cell phone number. When the user delivers message to need to be modified cell phone number saved in monolithic, we can write 
the cell phone number in the EEPROM, renew the user's cellular phone number. Even if drop electricity, this cell phone number can't throws to lose.

The function supported by the Q2403 is very complete, in our short message system, short message function is mainly used. The communication between monolithic and Q2403 implements receiving and sending function. The function of AT instruction includes:

1) Acquire the identification AT+CCID of SIM, checking up whether SIM card is inserted or not when the power of cell phone is turned on.

2) The short message sending instruction AT+CMGS; monolithic send short message to the user's cell phone When receiving alarming information.

3) Reading short message instruction AT+CMGR;A new cell phone number is got When the user delivers message to need to modified cell phone number saved at monolithic.

4) The short message deleting instruction AT+CMGD. Some useless short message is deleted after a short message is processed. So we can avoid SIM card save too many useless short message and avoid storage locations are wasted.

\section{Conclusion}

The design of hardware and software of our system and application program writing are introduced in this paper, we put it into practical application, obtained the effect of satisfaction. Once the small area appears a circumstance, the user's cell phone can receive alarming short message and life safety raised. If we make an appropriate modification to the software and hardware, our system can also be applied to monitor environment, the petroleum mine environment, monitoring farm crop growth condition, management system of traffic and safety defense system etc.

\section{Acknowledgment}

This project is supported by the Research Foundation of Education Bureau of Hunan Province, China(Grant No.10C1237).

\section{References}

[1]Xiaodong Chen. Temperature of serical communication based on monolithic. Modern electroc technology. Vol. 18 (2007), p. 59

[2]Chengjun He. C8051F monolithic system debug software based on serial port. Exerperience communication magazine. Vol. 10(2007), p.61

[3]Li yuan. Monolithic massage receiving and senging system based on.Q2403A. electricity design application. Vol. 83 (2007), p. 67

[4]WAVECOM company. Q2403 A and Q2403AProduct Specification. Version1.00[Z].2002.

[5]WAVECOM company. AT Commands Interface Guide. Version1.00[Z].2001. 